\title{
MRI PREDICTORS OF HEMORRHAGIC TRANSFORMATION IN 474 CONSECUTIVE STROKE PATIENTS TREATED BY IV THROMBOLYSIS
}

Rody EL NAWAR ${ }^{1,2}$, MD, Jennifer YEUNG ${ }^{1}$, MD, Julien LABREUCHE ${ }^{3}$, Marie Laure CHADENAT11, MD, Duc Long DUONG1 ${ }^{1}$, MD, Maxime DE MALHERBE ${ }^{4}, \mathrm{MD}$, Yves Sebastien CORDOLIANI ${ }^{5}, \mathrm{MD}$, Bertrand LAPERGUE, ${ }^{6,7}, \mathrm{MD}$, Fernando PICO ${ }^{1,7}, \mathrm{MD}, \mathrm{PHD}$

\author{
1 Department of Neurology and Stroke Center, Centre Hospitalier de Versailles, Versailles, France \\ ${ }^{2}$ Gilbert and Rose-Marie Chagoury School of Medicine, Lebanese American University, Beirut, Lebanon \\ Email : roudynawar@hotmail.com \\ 3Univ. Lille, CHU Lille, EA 2694 - Santé publique: épidémiologie et qualité des soins, F-59000 Lille, France. \\ ${ }^{4}$ Department of Radiology, Centre Hospitalier de Versailles, Versailles, France \\ ${ }^{5}$ Department of Radiology, Hopital Prive de Parly 2, Versailles, France \\ ${ }^{6}$ Department of Neurology and Stroke Center, Hopital Foch, Suresnes, France \\ 7Université Versailles Saint-Quentin en Yvelines et Paris Saclay
}

Background and Objective: Clinical and biological risk factors of hemorrhagic transformation (HT) after IV thrombolysis (IVT) have been well established in several registries such as SISTMOTS ${ }^{1}$. The added value of MRI parameters have been studied in small samples and are controversial2,4. Our objective is to evaluate the added value of MRI variables in HT in the above clinical and biological factors.

Methods: We enrolled between January 2011 and August 2017, a total of 474 consecutive patients with brain infarction (BI) proven by MRI and treated by IVT alone at our primary stroke center. Baseline demographic, clinical, biological and imaging characteristics were collected. MRI parameters were: volume of brain infarction in $\mathrm{cm}^{3}$ (B1000 diffusions, $A D C<500.10-6 \mathrm{~mm}^{2} / \mathrm{s}$ ), parenchymal Flair hyperintensity, hyperintense vessel sign, number of microbleeds, subcortical white matter hyperintensity (Fazekas scale) and thrombus length.

\section{Figure 1: Study flow chart}

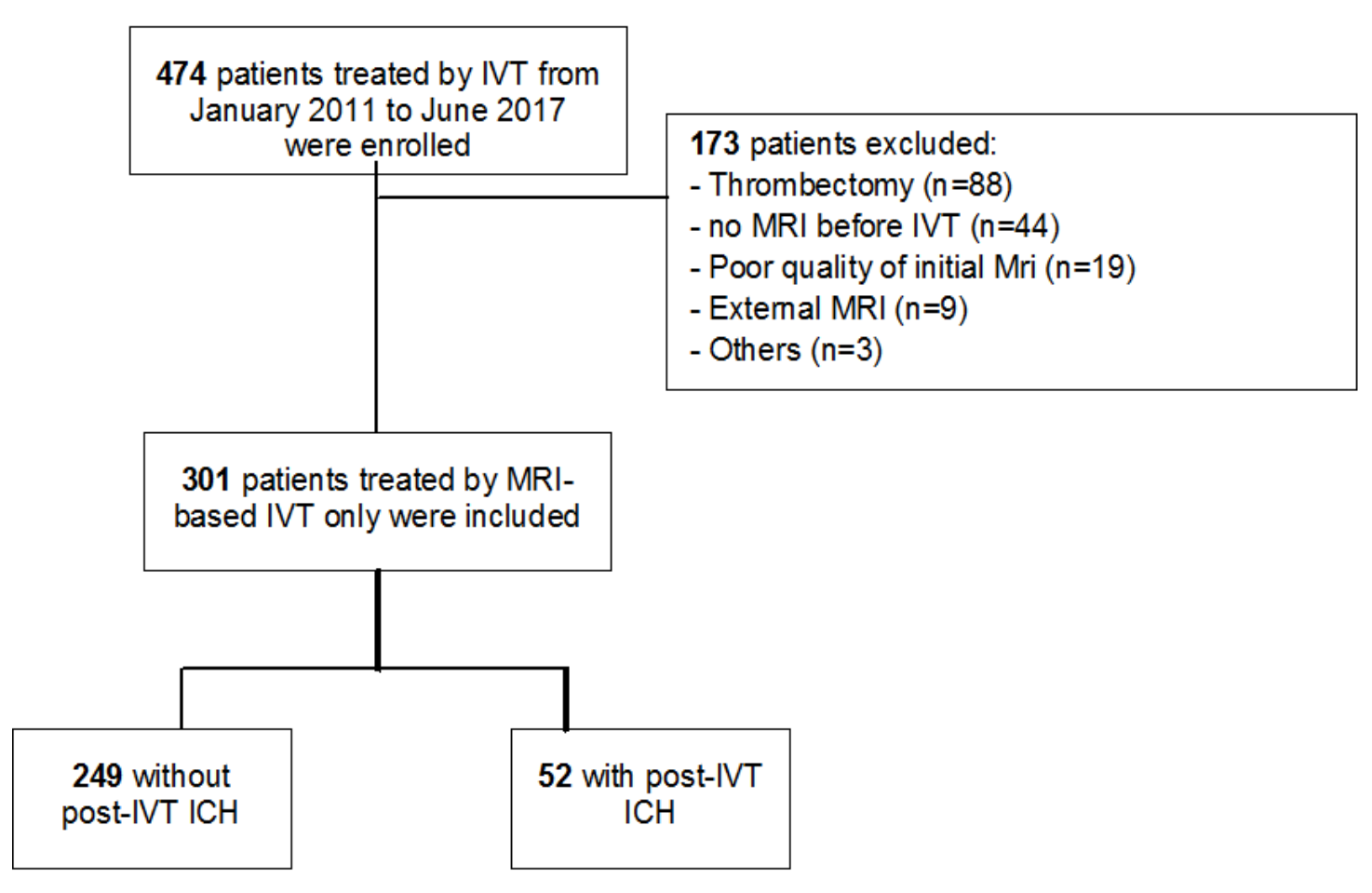

Abbreviations: $\mathrm{ICH}=$ intracranial hemorrhage; IVT=intravenous thrombolysis; $\mathrm{MRI}=$ magnetic resonance imaging
Results: 301 patients out of 474 were included (64\%). Main causes of exclusion were combined thrombectomy $(n=88)$ and no MRI before IVT $(n=44)$. In bivariate analyses, HT was significantly associated with the presence of more hyperintense vessel sign, thrombus length (> 8mm), higher $\mathrm{BI}$ volume (B1000 and $A D C<500)$. However, in multivariate analysis, only $\mathrm{BI}$ volumes were significantly associated with $H T$. The discrimination value of the multivariable model including both DWI volume and clinical model (AUC, $0.80 ; 95 \% \mathrm{Cl}, 0.74$ to 0.86 ) was significantly improved compared to the model based only on clinical variables $(p=0.012)$ (i.e. age and NIHSS)

Conclusions: Brain infarction volume on DWI appears to be the only predictive MRI parameter adding value to the clinicobiological parameters in $\mathrm{HT}$.

Figure2: Receiver operating characteristic (ROC) curves for prediction of post-thrombolysis ICH by clinical model and MRI parameters.
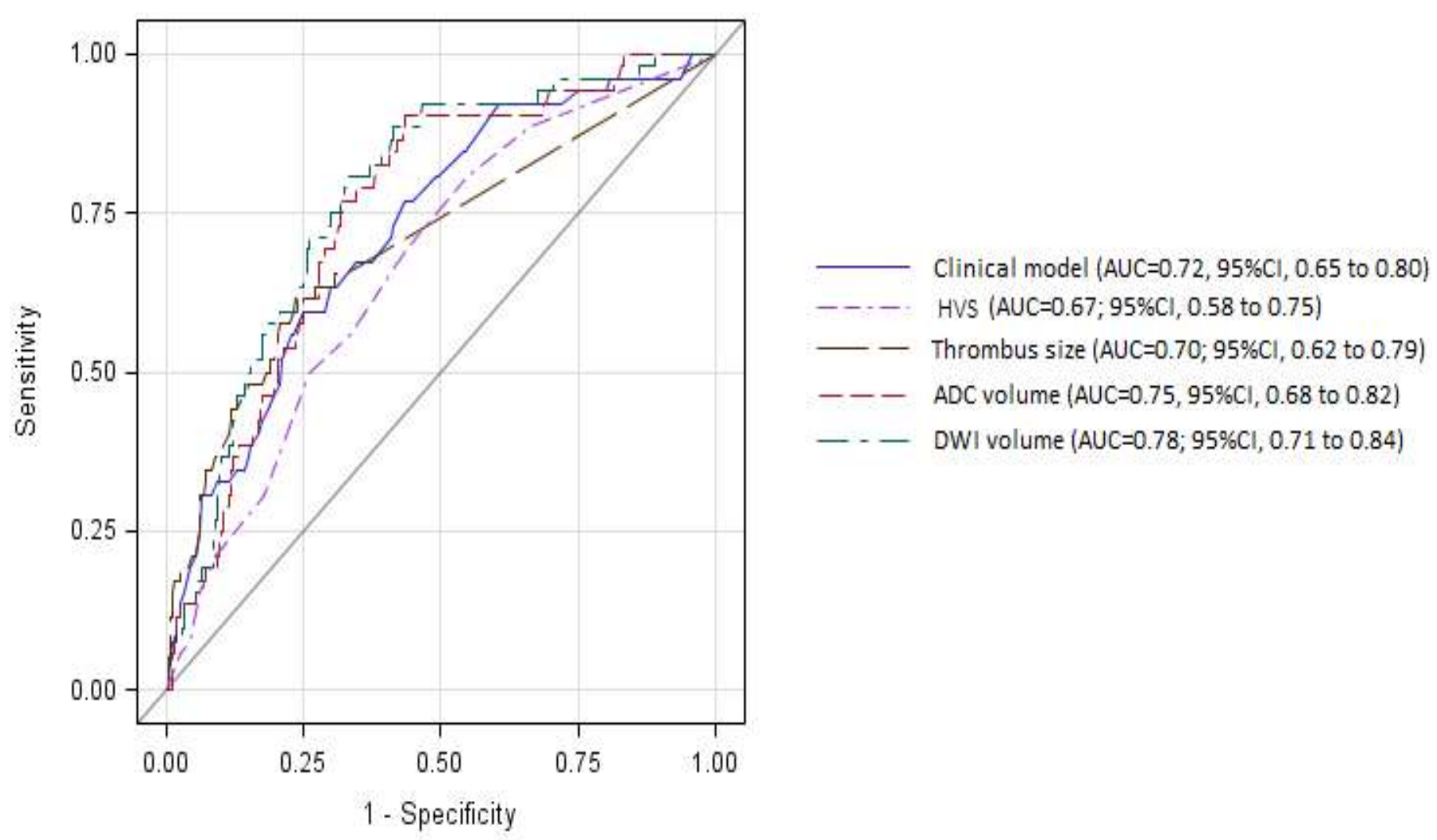

Abbreviations: $A D C=A p p a r e n t$ Diffusion Coefficient, $A U C=$ area under the curve, $\mathrm{DWI}=$ diffusion weighted imaging, $\mathrm{ICH}=$ intracerebral hemorrhage

Corresponding author: Fernando PICO, MD, PhD FPico@ch-versailles.fr

Department of Neurology and Stroke Center,Centre Hospitalier de Versailles, Versailles, France177 Rue de Versailles, 78150 Le Chesnay Université Versailles Saint-Quentin en Yvelines et Paris Saclay

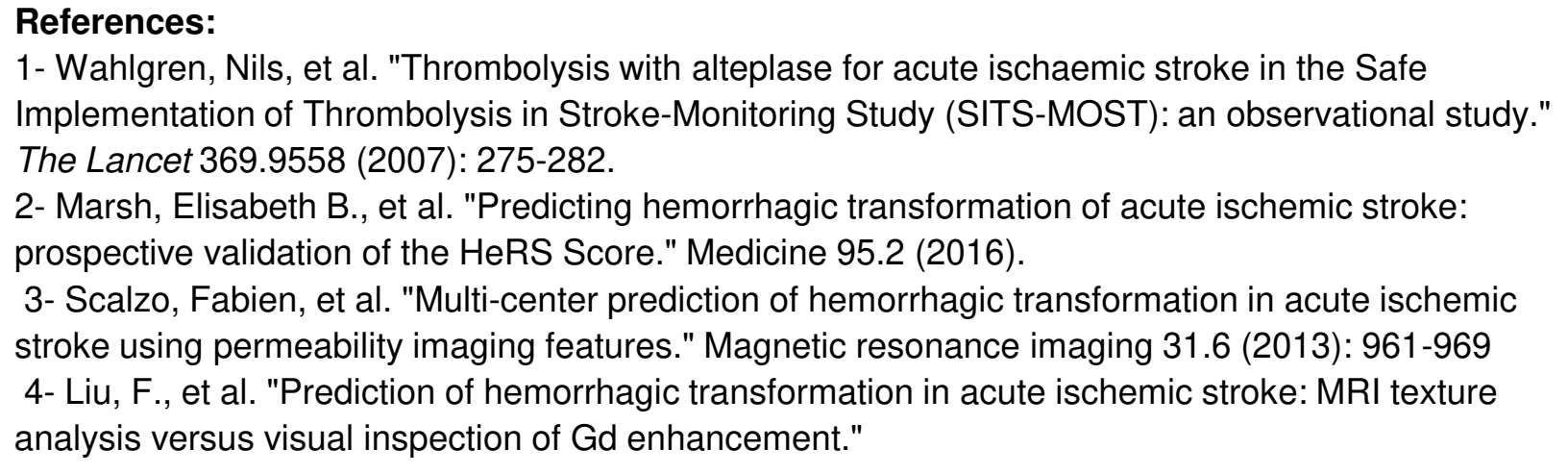

